\title{
T1-weighted Black-blood Magnetic Resonance Imaging Helps to Stage Thrombus on Segment Level and Predict the Outcome of Endovascular Therapy in Patients with Cerebral Venous Sinus Thrombosis
}

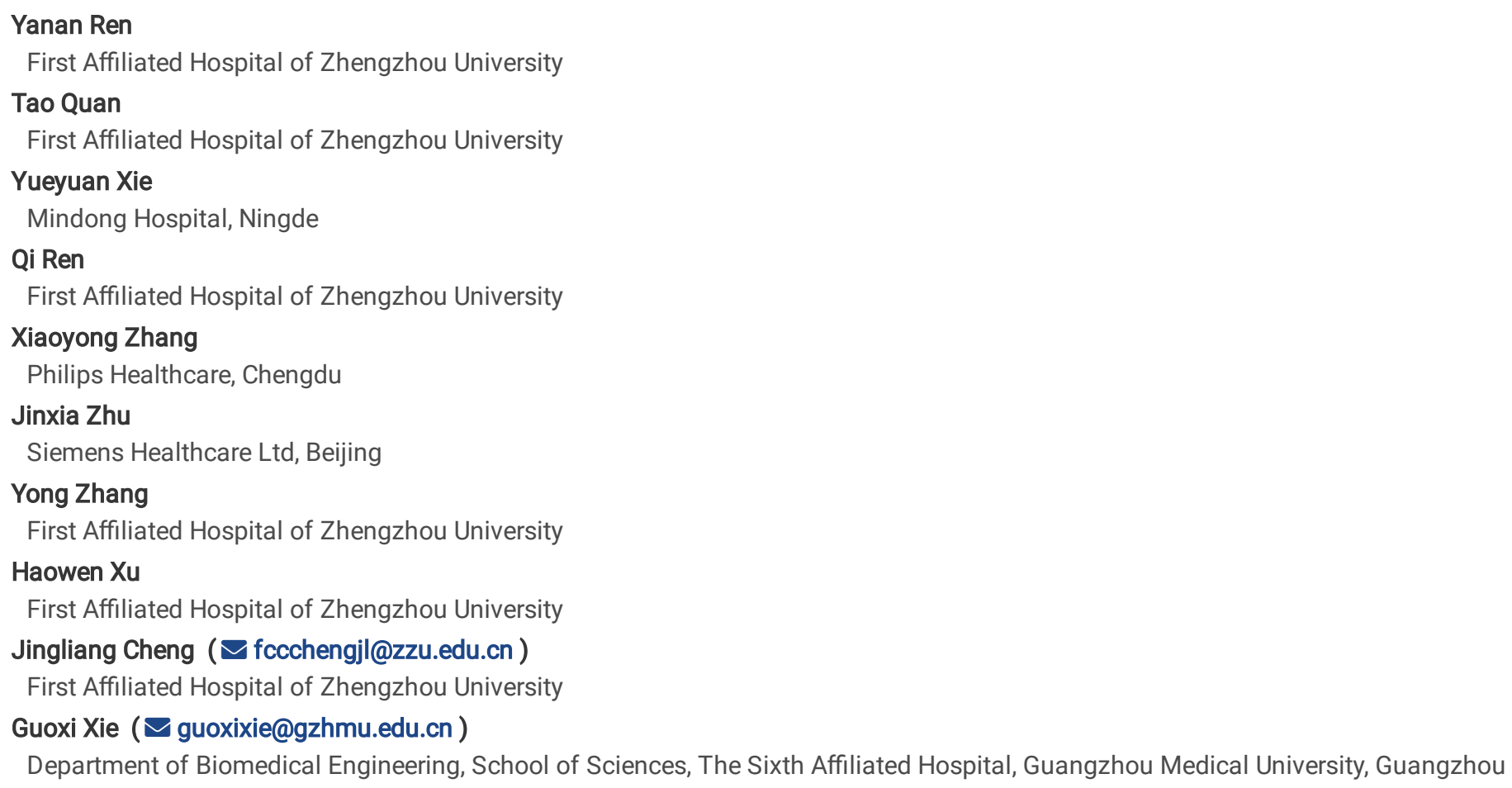




\section{Abstract \\ Purpose}

Clinical approach for staging cerebral venous sinus thrombosis (CVST) depends on the duration from symptom onset to clinical diagnosis, which is subjective and may be incorrect. To investigate if T1-weighted signal characteristics of the thrombus can be used for staging the thrombus on segment level and predicting endovascular therapy outcome.

\section{Methods}

Ninety-one CVST patients underwent non-contrast-enhanced T1-weighted 3D variable-flip-angle fast-spin-echo (T1-SPACE) and 29 of them received endovascular therapy. We divided the 91 patients into acute, subacute, and chronic CVST stage according to the clinical approach, and then analyzed the T1 weighted signal characteristics (i.e., thrombus shape and signal intensity) of the thrombus at each stage. To investigate the endovascular therapy outcome associated with the thrombus stage and signal characteristics, we evaluated the thrombolytic ratio on patient level and recanalization rate on segment level, respectively.

\section{Results}

The thrombi at acute or chronic stage were depicted as isointense signals but as hyperintense signals at subacute stage. Compared to the thrombus at acute stage, the thrombus at chronic stage was contractive and can be distinguished. Developing thrombi at transition periods of acute-to-subacute or subacute-to-chronic stage were found appearing mixed iso-/hyper-intense signals. On patient's level, the patients at earlier stage had higher thrombolytic ratio (acute: $80.7 \pm 18.3 \%$, subacute: $62.6 \pm 36.3 \%$, chronic: $22.9 \pm 24.2 \%$ ). On segment level, the thromboembolic segments of isointense or mixed iso-/hyper-intense thrombi at non-chronic stage had good thrombolytic outcome.

\section{Conclusion}

T1-weighted signal characteristics of the thrombus can be used for staging CVST and predicting the endovascular therapy outcome.

\section{Introduction}

Cerebral venous sinus thrombus (CVST) is usually affecting young individuals, representing $0.5-1.0 \%$ of all strokes [1, 2]. Despite increased advances in recognition of CVST in recent years, accurate and personalized treatment of CVST remains a difficult task in clinical practice because the thrombus composition changes during CVST progress [3]. Recent reports demonstrated that the outcome of endovascular therapy for patients with CVST is associated with the thrombus stage [4-6]. Therapeutic schedules for CVST should be based on correct estimation of the thrombus stage [7-9]. However, current clinical methods for staging the thrombus only depends on the duration from symptom onset to clinical diagnosis, which is subjective and may be incorrect because CVST can be asymptomatic $[8,10]$.

Both animal and clinical studies demonstrated that magnetic resonance (MR) T1 relaxation time of the thrombus is related to the thrombus methemoglobin formation and further processing [11-13]. When erythrocytes lyse, the iron of the thrombus is oxidized and converted to paramagnetic $\mathrm{Fe}^{3+}$; the accumulation of paramagnetic $\mathrm{Fe}^{3+}$ shortens the thrombus $\mathrm{T} 1$ relaxation time. After the removal of $\mathrm{Fe}^{3+}$ by macrophages, the thrombus T1 relaxation time is then prolonged. Thus, the change in the thrombus T1 relaxation time leads to the variation of thrombus signal intensities on T1-weighted MR images [14]. Therefore, thrombus signal characteristics on T1-weighted MR images may help to determine the thrombus stage and thus can be used to predict the CVST treatment outcome.

Recently, a black-blood MR technique, non-contrast-enhanced T1-weighted 3D variable-flip-angle fast-spin-echo (T1-SPACE), was introduced to detect CVST [15]. With the blood flow signal suppressed by the inherent black-blood effect of T1-SPACE, the intraluminal thrombus signal characteristics can be visualized and analyzed without the mimic artifacts of blood flows $[15,16]$. Thus, we hypothesized that the thrombus signal characteristics on T1-SPACE can be used to determine the thrombus stage on segment level and helps for predicting the endovascular therapy outcome of CVST patients.

\section{Materials And Methods}

\section{Patients and characteristics}


This retrospective study was performed on 91 patients who were diagnosed with CVST at a hospital from May 2016 to Dec 2020 . The study was approved by the Scientific Research and Clinical Trial Ethics Committee of the First Affiliated Hospital of Zhengzhou University and was exempt from informed consent due to the purely retrospective analysis. Inclusion criteria included: confirmation of CVST, the interval from the first clinical symptom onset to MR examination and endovascular therapy within 30 days; Exclusion criteria included general contraindications to MR examination and medical history of CVST or other synchronous brain diseases. Recurrent CVST patients were also excluded because residual thrombi may exist $[17,18]$.

Patients' information, including age, gender, duration of the clinical symptom onset, MR imaging results, DSA images, risk factors, and therapy methods, were collected for analysis. Based on the interval from the clinical symptom onset to MR examination, the patients were divided into three groups: acute stage ( $\leq 7$ days), subacute stage (8 to 15 days), and chronic stage (16 to 30 days). The patients' characteristics were listed in Table 1.

\section{MR imaging}

All CVST patients were conducted by T1-SPACE on a 3.0-T MR scanner (MAGNETOM Prisma, Siemens Healthcare, Erlangen, Germany) with a 64-channel head $/$ neck coil. The scan parameters included: repetition time $=650 \mathrm{~ms}$, echo time $=13 \mathrm{~ms}$, parallel acceleration factor $=2$, turbo factor $=40$, bandwidth $=539 \mathrm{~Hz} /$ pixel, field of view $=260 \times 208 \mathrm{~mm}^{2}$, voxel size $=0.8 \times 0.8 \times 0.8 \mathrm{~mm}^{3}$, acquisition time $=5 \mathrm{~min} 27 \mathrm{~s}$.

The MR examination was performed before endovascular therapy for the patients within three days. One patient received anticoagulation initially and transferred to endovascular therapy later because the symptoms unrelieved and suddenly deteriorated. Thus, the patient was conducted by T1-SPACE three times: 1 . before anticoagulation; 2 . after the anticoagulation 2 days but before endovascular therapy; 3 . followup study after endovascular therapy.

\section{Treatments of CVST}

Conventional anticoagulation with low molecular weight heparin (LMWH) was the initial treatment in all the patients after MR examination confirming the CVST. Patients who satisfied the following criteria would receive endovascular thrombectomy and thrombolysis: 1 . Glasgow Coma Score (GCS) was low than 10 points at admission or during treatment; 2 . symptoms were rapidly deteriorated after anticoagulation treatment (GCS < 10 points); 3. straight sinus thrombosis; 4. large area cerebral infarction or intracranial hematoma. The exclusion criteria were as follows: 1. anticoagulant therapy was effective and the symptoms significantly relieved; 2 . significant cardiac, liver, or renal dysfunction; 3. patients with a cerebral hernia; 4. CVST secondary to malignancy such as leukemia; 5 . patients refused interventional thrombolysis.

LMWH (85IU/Kg, SANOFI, CHINA) was given twice per day for 14 days for patients who received conventional anticoagulant treatment. Partial thromboplastin time (PTT) monitoring was performed. Oral anticoagulation combined before the discontinuation of LMWH.

Twenty-nine of 91 patients received endovascular therapy. The procedures were initially performed under local anesthesia or monitored awake sedation. Femoral arterial and venous access were chosen, and a bolus dose of 3000 to 5000 units of heparin was given at the beginning. A cerebral arterial angiogram diagnosed CVST firstly, and an $8 \mathrm{~F}$ guide sheath or guiding-catheter was then advanced into the carotid vein. Machine thrombectomy was performed as the first-line endovascular therapy with either stent-retriever (Solitaire stent, Medtronic, USA) or direct contact aspiration technique by an aspiration catheter (Naive catheter, Medtronic, USA). After several attempts to retrieve the intra-sinus thrombus, a microcatheter was placed in the superior sagittal sinus or the straight sinus. Urokinase (15000U/Kg, iv, qd) was administered by microcatheter. The microcatheter was remained until significant clinical improvement or partial recanalization of the sinus confirmed by DSA.

\section{MR image analysis}

The acquired MR images were loaded onto a workstation (Leonardo, Siemens AG, Germany) for image review and analysis. The cerebral venous system was divided into 13 segments for analysis, i.e., superior sagittal sinus, inferior sagittal sinus, right transverse sinus, right sigmoid sinus, left transverse sinus, left sigmoid sinus, straight sinus, confluences of sinus, veins of Galen, internal cerebral veins, Rosenthal veins, veins of Labbé, and cortical veins.

To determine which venous segment occurs thrombosis, the presence of thrombus in each segment as detected by T1-SPACE was recorded and reviewed by two experienced radiologists, both blinded to the patients' clinical information, each with $>5$ years MR experience.

Discrepancies were resolved by two readers using consensus with the auxiliary DSA and/or other MR images.

To quantitatively analyze the thrombus signal characteristics on T1-SPACE images, the thrombus was semi-automatically segmented, and then the volume and signal intensities of the thrombus were measured from T1-SPACE images using an open software (ITK-

SNAP, www.itksnap.org/pmwiki/pmwiki.php). Due to the use of parallel imaging in T1-SPACE, the contrast ratio (CR), instead of contrast to 
noise ratio, was calculated to quantify the contrast between two tissues [16]. Specifically, the $\mathrm{CR}_{\mathrm{tg}}$ was the ratio of signal intensities between the thrombus and gray matter and $\mathrm{CR}_{\mathrm{tl}}$ was the ratio of signal intensities between the thrombus and venous lumen.

\section{Outcome of the endovascular therapy}

To investigate if the thrombus signal characteristics on T1-SPACE images can be used to predict the endovascular therapy outcome, we collected all DSA images before and after the endovascular therapy. The recanalization status was then evaluated on patient and segment levels, respectively. The evaluation was finished by two interventional neurologists with blinded to the randomization assignments. Both neurologists have more than 5 years of interventional experience. Any discrepancies between the neurologists were resolved by consensus.

Similar to the method introduced by Porter and Moneta [19], thrombolytic ratio was used to evaluate the recanalization status on the patient level. The thrombolytic ratio was calculated using ([total thrombus scores pre-lysis] - [total thrombus scores post-lysis]) / [total thrombus scores pre-lysis] $\times 100 \%$, where a thrombus score of each segment was calculated based on a 4-point scale: $0=$ patent and completely free of thrombus; 1 = partial occlusion (<50\%); 2 = partial occlusion $(>50 \%) ; 3=$ complete occlusion, and thus the total thrombus scores before and after lysis were calculated by adding the scores of the thromboembolic segments.

According to the method introduced by Aguiar et al [20], the following standard was used to assess the recanalization status on the segment level. The standard includes complete recanalization, partial recanalization, and absent recanalization. 1. complete recanalization defined as blood flow without any interruption; 2. partial recanalization defined as small interruptions of continuous blood flow and narrowing of the venous lumen; 3. absent recanalization defined as interrupted blood flow. After all the thromboembolic segments were assessed, the recanalization rate was then calculated as the number of recanalization segments divided by the total number of all thromboembolic segments.

\section{Statistical analysis}

Statistical analysis was performed using SPSS 21.0 (IBM, USA). Continuous variables following a normal distribution and equal variances were presented as means $\pm S D$ and analyzed using the one-way ANOVA; otherwise, they were presented as median (interquartile range) and analyzed using Kruskal-Wallis $H$ test. $L S D$ - $t$ test was used for further comparison between groups of the continuous variables after the oneway ANOVA analysis. Mann-Whitney $U$ test was used for further comparison between groups for abnormal distribution variables. Chi-squared test was used to compare categorized variables at different stages. The interobserver agreements in thrombus presence of all patients, thrombus scores, and recanalization status were evaluated by Cohen's kappa coefficient. A value of $p<0.05$ was considered significant.

\section{Results}

\section{MR imaging}

All patients successfully conducted the MR examination. Based on the interval from symptom onset to the MR examination, 25 (27.5\%) patients were at acute stage, 31 (34.1\%) patients were at subacute stage, and 35(38.4\%) were at chronic stage. A total of 378 thromboembolic segments, including 107 segments in the acute patients, 142 segments in the subacute patients, and 129 segments in the chronic patients, were identified on T1-SPACE images. The thromboembolic segments included 58 the superior sagittal sinus, 3 inferior sagittal sinus, 21 straight sinus, 44 confluences of sinuses, 48 left transverse sinus, 38 left sigmoid sinus, 56 right transverse sinus, 47 right sigmoid sinus, 10 veins of Galen, 7 internal cerebral veins, 3 Rosenthal vein, 4 veins of Labbé, and 40 cortical veins. Excellent interobserver agreement on identifying the thromboembolic segments was obtained by T1-SPACE $(K=0.902, p<0.001)$.

Representative images from acute, subacute, and chronic patients were demonstrated in Fig. 1. The thrombi at acute (a) or chronic (c) stage were typically depicted as isointense signals but as hyperintense signals at subacute (b) stage. Compared to the isointense thrombus at the acute stage, the isointense thrombus at the chronic stage is contractive and can be distinguished. Mixed iso-/hyper-intense thrombus that coexisted isointense and hyperintense signals was also found on some patients (Fig. 2). These patients can be at acute (a), subacute (b), or chronic (c) stage according to the interval from the clinical symptom onset to MR examination.

Quantitative analysis results of the thrombus signal characteristics were shown in Table 2. The $\mathrm{CR}_{\mathrm{tg}}$ and $\mathrm{CR}_{\mathrm{tl}}$ of patients at subacute stage were significantly higher than those of patients at acute or chronic stages. The thrombus volume among patients at different stages was not significantly different. 


\begin{tabular}{|lll|}
\hline parameters & T1-SPACE & PC-MRV \\
\hline Repetition time, ms & 650 & 56.25 \\
\hline Echo time, ms & 13 & 9.89 \\
\hline Inversion time, ms & N/A & N/A \\
\hline Flip angle, & N/A & 15 \\
\hline GRAPPA & 2 & 2 \\
\hline Turbo factor & 40 & N/A \\
\hline Bandwidth, Hz/pixel & 539 & 300 \\
\hline Field of View, mm ${ }^{2}$ & $260 \times 208$ & $220 \times 206$ \\
\hline Voxel size, mm ${ }^{3}$ & $0.8 \times 0.8 \times 0.7$ & $0.9 \times 0.9 \times 1.0$ \\
\hline Acquisition time & 5 min $27 \mathrm{~s}$ & 7 min $29 \mathrm{~s}$ \\
\hline Note: N/A = Not Applicable & \\
\hline
\end{tabular}

Outcome Of Endovascular Therapy

Among the patients received endovascular therapy, 8 of them were at acute stage, 10 of them were at subacute stage, and 11 of them were at chronic stage. The thrombolytic ratio of the patients at acute, subacute, and chronic stages were $(80.7 \pm 18.3) \%,(62.6 \pm 36.3) \%$, and $(22.9 \pm 24.2) \%$, respectively. The thrombolytic ratio was associated with the stage on patient level $(p<0.001)$.

The recanalization rates were shown in Table 3. Specifically, all thromboembolic segments in acute patients were complete or partially recanalized. None segment in the acute patients was in absent recanalization. In contrast, $18.2 \%$ of the thromboembolic segments in subacute patients and $46.3 \%$ of the thromboembolic segments in chronic patients were in absent recanalization. The acute patients achieved the best thrombolytic results based on the analysis of segment level. Recanalization status on segment level was significantly different among the three stages $(p<0.001)$. 
Table 3

Recanalization rates of the thromboembolic segments of CVST patients received endovascular therapy

\begin{tabular}{|llll|}
\hline & Acute stage & $\begin{array}{l}\text { Subacute } \\
\text { stage }\end{array}$ & Chronic stage \\
\hline $\begin{array}{l}\text { Complete } \\
\text { recanalization }\end{array}$ & $(22 / 30) 73.3 \%$ & $(19 / 33) 57.6 \%$ & $(3 / 41) 7.3 \%$ \\
\hline Partial recanalization & $(8 / 30) 26.7 \%$ & $(8 / 33) 24.2 \%$ & $(19 / 41) 46.3 \%$ \\
\hline Absent recanalization & $(0 / 30) 0.0 \%$ & $(6 / 33) 18.2 \%$ & $(19 / 41) 46.3 \%$ \\
\hline
\end{tabular}

It is interesting that if the thrombi in patients were further divided into isointense signal type, mixed iso-/hyper-intense signal type, and hyperintense signal type, the recanalization of different types of thrombi was found significantly different based on segment level analysis (Table 4). For the patients at acute CVST stage, the recanalization of the isointense thrombus was comparable to the mixed iso-/hyper-intense one. For the patients at subacute CVST stage, the recanalization of the isointense and mixed iso-/hyper-intense thrombi was better than the hyperintense one. For the patients at chronic stage, the thrombus was not recanalized well. 
Table 4

Recanalization analysis of the thromboembolic segments based on thrombus signal characteristics

\begin{tabular}{|lllll|}
\hline & Recanalization & $\begin{array}{l}\text { Isointense } \\
\text { thrombus }\end{array}$ & $\begin{array}{l}\text { Mixed } \\
\text { iso-/hyper- } \\
\text { intense } \\
\text { thrombus }\end{array}$ & $\begin{array}{l}\text { Hyperintense } \\
\text { thrombus }\end{array}$ \\
\hline $\begin{array}{l}\text { Acute } \\
\text { stage }\end{array}$ & Complete & $(14 / 18) 77.8 \%$ & $(8 / 12) 75.0 \%$ & N/A \\
\hline & Partial & $(4 / 18) 22.2 \%$ & $(4 / 12) 33.3 \%$ & N/A \\
\hline $\begin{array}{l}\text { Subacute } \\
\text { stage }\end{array}$ & Absent & $(0 / 18) 0.0 \%$ & $(0 / 12) 0.0 \%$ & N/A \\
\hline & Partial & $(2 / 6) 33.3 \%$ & $(2 / 11) 18.2 \%$ & $(4 / 16) 25.0 \%$ \\
\hline $\begin{array}{l}\text { Chronic } \\
\text { stage }\end{array}$ & Absent & $(0 / 6) 0.0 \%$ & $(1 / 11) 9.1 \%$ & $(5 / 16) 31.3 \%$ \\
\hline & Complete & $(1 / 30) 3.3 \%$ & $(2 / 11) 18.2 \%$ & N/A \\
\hline & Partial & $(17 / 30) 56.7 \%$ & $(2 / 11) 18.2 \%$ & N/A \\
\hline Note: N/A $=$ Not Applicable & & $(12 / 30) 40.0 \%$ & $(7 / 11) 63.6 \%$ & N/A \\
\hline
\end{tabular}

Representative T1-SPACE and DSA images of patients from acute to chronic stages were shown in Fig. 3 . As expected, the patient (A) at acute stage with isointense thrombus was completely recanalized. The patient (C) at subacute stage with hyperintense thrombus and the patient (E) at chronic stage with isointense thrombus were not recanalized well. The patient at subacute stage (B) with mixed iso-/hyper-intense thrombus were completely recanalized. In contrast, although the thrombus (D) at the chronic stage also coexisted isointense and hyperintense signals, the dissolution failed.

More interestingly, thrombus development was found in the patient who conducted the MR scan three times. Representative images of the patient were shown in Fig. 4. Thrombus (yellow arrow on part A) with hyperintense signals 


\section{was found in the transverse sinus on the first MR scanning on the 11th day since symptom onset. And there was only a tiny thrombus with isointense signals (red arrow on part A) within the superior sagittal sinus meanwhile. Two days later, the thrombus developed and fulfilled the whole superior sagittal sinus. The newly developing thrombus (white arrow on part B) was thus definitely at acute stage. As expected, the acute thrombus was depicted as isointense signals on T1- SPACE images. After endovascular therapy, the acute thrombus was successfully dissolved (blue arrow on part C) while the thrombus with hyperintense signals was residual (yellow arrow on part C), confirming that the isointense thrombus at acute stage was easier to dissolve than the hyperintense thrombus at subacute stage. This also demonstrated that the thrombus stage on segment level can be determined by using the thrombus T1-weighted signal characteristics.}

\section{The interobserver reproducibility for thrombus scores and recanalization status had $K$ value of 0.814 and 0.866 , respectively.}

\section{Discussion}

In this study, the T1-weighted MR signal characteristics of the thrombi at different stage were analyzed. Our experiment results demonstrated that the signal characteristics can be used for staging CVST on segment level and predicting the outcome of endovascular therapy.

Different signal characteristics of acute, subacute, and chronic thrombi were found on T1-SPACE images. T1-SPACE uses thrombus as the imaging target and suppresses the interfering venous blood signals, making the thrombus directly visualized [15]. As the subacute thrombus is rich in short-T1 methemoglobins, the thrombus thus appears hyperintense signals on T1-SPACE images [12, 15]. Meanwhile, as the venous blood flow signals are suppressed, T1-SPACE is also sensitive in detecting isointense thrombus at acute and chronic stages. Although the acute and the chronic thrombi have very similar signal intensities on T1-SPACE images, they can be distinguished by the thrombus features on T1-SPACE images. Specifically, the thrombus at the acute stage is newly formed, whereas the emboli shapes are smooth and homogeneous. In contrast, the thrombus at the chronic stage is organized and contractive. Thus, the thrombus at different stages has different signal characteristics on T1-SPACE images. This makes determining the thrombus stage on segment level possible.

CVST is a developing process with the thrombus composition changing [21]. Thrombus signal characteristics at transition period are more complicate than those at typical acute, subacute, and chronic stages on T1-SPACE images. As the thrombus at acute or chronic presented in isointense signals but as hyperintense signals at subacute stage, the developing thrombi at the transition periods from acute to subacute or from the subacute to chronic stage should coexist isointense and hyperintense signals. Nevertheless, the signal characteristic differences between the thrombi at these two transition periods were found on T1-SPACE images. At the transition period from the acute to subacute stage, the thrombus has isointense signals in the thrombus center surrounded by hyperintense signals within the venous lumen. In contrast, the thrombus has hyperintense signals in the thrombus center surrounded by isointense signals at the transition period from the subacute to chronic stage. These findings are in accordance with the mechanism of thrombus development that the thrombus first occurs adhering to the venous vessel wall and then spreads to the center (Fig. 4). Thus, T1-SPACE can be used to find the thrombus at the transition period, which makes the thrombus staging more accurately and objectively.

The thrombus stage was found to be associated with recanalization of endovascular therapy. As the obstructions in the venous lumen, thrombus formation is the source of thrombus $\mathrm{T} 1$ relaxation time at different stages and its recanalization status after endovascular therapy $[5,11]$. The fresh thrombus is loose and rich in deoxyhemoglobins at acute and acute-to-subacute stages [22, 23]. Thus, thrombus removal and recirculation can be achieved. When the thrombus progresses to the subacute stage, it is rich in short-T1 methemoglobins and thus appears hyperintense signals on T1-SPACE images, which may be a sign of endovascular therapy. At the subsequent subacute-to-chronic and chronic stages, the hyperintense thrombi revert to isointense signals primarily with the methemoglobins transferred gradually [22]. The increasing collagen and cross-linked fibrin make the destruction of thrombi more difficult [24]. Such thrombolytic difference during thrombus process was verified in this work (Table 4). Especially for the patient shown in Fig. 4, the acute thrombus in the superior sagittal sinus was dissolved entirely, but the subacute thrombus in the right transverse sinus maintained residual. Therefore, the thrombus signal characteristics on T1SPACE can be used to predict the outcome of endovascular therapy for each thromboembolic segment.

Page 8/13 
Our experiment results are in agreement with a previous study [5]. The previous study reported that complete recanalization rates of endovascular treatment for acute CVST patients with isointense and hyperintense thrombi were $84.6 \%$ and $29.6 \%$ using residual luminal narrowing $<50 \%$ as complete recanalization criterion. In our study, the thromboembolic segments with isointense thrombus also have much better thrombolytic outcomes than those with hyperintense thrombus in non-chronic CVST patients (Table 4). Apart from this, a new finding in our study is that the CVST patients may not only have isointense or hyperintense thrombus but also have mixed iso-/hyper-intense one. The mixed iso-/hyper-intense thrombus at the transition period from acute to subacute stage also have good thrombolytic results.

\section{Limitations}

The work has at least a limitation that the follow-up study was not carried out yet. As the complex cerebral venous system and strong collateral circulation could affect the CVST treatment outcome, tracking the treatment effect to discharge is far from enough. Long-term follow-up study should be performed to evaluate the recanalization and restoration status of cerebral venous vessels in the future study.

\section{Conclusions}

The T1-weighted thrombus signal characteristics can be used for determining the thrombus stage on segment level and predicting the thrombolytic outcome of endovascular therapy for CVST patients. T1-SPACE could be a potential and valuable technique for the diagnosis and prognosis of CVS.

\section{Declarations}

Funding: This work was supported by the National Natural Science Foundation of China [grant numbers: 81971607].

Conflicts of interest/competing interests: The authors declare that they have no conflict of interest.

Availability of data and material: The data used during the current study are available from the corresponding author on reasonable request.

Code availability: Not applicable.

Authors' contributions: Yanan Ren: All authors contributed to the study conception and design. Material preparation, data collection and analysis were performed by Qi Ren and Yueyuan Xie. The first draft of the manuscript was written by Yanan Ren. The methodology was supported by Xiaoyong Zhang and Jinxia Zhu. The formal analysis was completed by Yong Zhang, Jingliang Cheng, Tao Quan and Haowen

Xu. The final draft of the manuscript was reviewed and edited by Guoxi Xie. All authors read and approved the final manuscript.

Ethics approval: Approval was granted by the Scientific Research and Clinical Trial Ethics Committee of the First Affiliated Hospital of Zhengzhou University (SS-2018-11) and was exempt from informed consent due to the purely retrospective analysis.

Acknowledgments: None

\section{References}

1. Stam, J. Thrombosis of the cerebral veins and sinuses. N Engl J Med, 352, 1791-1798 https://doi.org/10.1056/NEJMra042354 (2005).

2. Ferro, J. M. \& Aguiar de Sousa, D. Cerebral Venous Thrombosis: an Update. Curr Neurol Neurosci Rep, 19, 74 https://doi.org/10.1007/s11910-019-0988-x (2019).

3. Silvis, S. M., de Sousa, D. A., Ferro, J. M. \& Coutinho, J. M. Cerebral venous thrombosis. Nat Rev Neurol, 13, 555-565 https://doi.org/10.1038/nrneurol.2017.104 (2017).

4. Capecchi, M., Abbattista, M. \& Martinelli, I. Cerebral venous sinus thrombosis. J Thromb Haemost, 16, 1918-1931 https://doi.org/10.1111/jth.14210 (2018).

5. Yang, X. X. et al. Predictors of successful endovascular treatment in severe cerebral venous sinus thrombosis. Annals of Clinical and Translational Neurology, 6, 755-761 https://doi.org/10.1002/acn3.749 (2019).

6. Karanam, L. S. et al. Local Intrasinus Thrombolysis for Cerebral Venous Sinus Thrombosis. J Vasc Interv Neurol, 9, 49-54 (2016).

7. Ferro, J. M. et al. European Stroke Organization guideline for the diagnosis and treatment of cerebral venous thrombosis - endorsed by the European Academy of Neurology. Eur J Neurol, 24, 1203-1213 https://doi.org/10.1111/ene.13381 (2017).

8. Saposnik, G. et al. Diagnosis and management of cerebral venous thrombosis: a statement for healthcare professionals from the American Heart Association/American Stroke Association., 42, 1158-1192 https://doi.org/10.1161/STR.0b013e31820a8364 (2011). 
9. Fan, Y. et al. Chinese Stroke Association guidelines for clinical management of cerebrovascular disorders: executive summary and 2019 update of clinical management of cerebral venous sinus thrombosis. Stroke Vasc Neurol, 5, 152-158 https://doi.org/10.1136/svn-2020000358 (2020).

10. Benjamin, C. G. et al. Postoperative cerebral venous sinus thrombosis in the setting of surgery adjacent to the major dural venous sinuses. Journal of Neurosurgery, 131, 1317-1323 https://doi.org/10.3171/2018.4.Jns18308 (2019).

11. Saha, P. et al. Magnetic resonance T1 relaxation time of venous thrombus is determined by iron processing and predicts susceptibility to lysis., 128, 729-736 https://doi.org/10.1161/CIRCULATIONAHA.113.001371 (2013).

12. Xie, G. et al. Black-blood thrombus imaging (BTI): a contrast-free cardiovascular magnetic resonance approach for the diagnosis of nonacute deep vein thrombosis. J Cardiovasc Magn Reson, 19, 4 https://doi.org/10.1186/s12968-016-0320-8 (2017).

13. Chen, $\mathrm{H}$. et al. Cardiovascular magnetic resonance black-blood thrombus imaging for the diagnosis of acute deep vein thrombosis at 1.5 Tesla. J Cardiovasc Magn Reson, 20, 42 https://doi.org/10.1186/s12968-018-0459-6 (2018).

14. Ghoneim, A., Straiton, J., Pollard, C., Macdonald, K. \& Jampana, R. Imaging of cerebral venous thrombosis. Clin Radio, 75, 254-264 https://doi.org/10.1016/j.crad.2019.12.009 (2020).

15. Yang, Q. et al. Early Detection and Quantification of Cerebral Venous Thrombosis by Magnetic Resonance Black-Blood Thrombus Imaging., 47, 404-409 https://doi.org/10.1161/STROKEAHA.115.011369 (2016).

16. Wang, G. et al. Cerebral Venous Thrombosis: MR Black-Blood Thrombus Imaging with Enhanced Blood Signal Suppression. AJNR Am J Neuroradiol, 40, 1725-1730 https://doi.org/10.3174/ajnr.A6212 (2019).

17. Palazzo, P. et al. Venous Thrombotic Recurrence After Cerebral Venous Thrombosis: A Long-Term Follow-Up Study., 48, $321-326$ https://doi.org/10.1161/STROKEAHA.116.015294 (2017).

18. Martinelli, I. et al. Long-term evaluation of the risk of recurrence after cerebral sinus-venous thrombosis., 121, 2740-2746 https://doi.org/10.1161/CIRCULATIONAHA.109.927046 (2010).

19. Porter, J. M. \& Moneta, G. L. Reporting standards in venous disease: an update (1995) International Consensus Committee on Chronic Venous Disease.J Vasc Surg21:635-45. 10.1016/s0741-5214(95)70195-8.

20. Aguiar de Sousa, D., Lucas Neto, L., Canhao, P. \& Ferro, J. M. Recanalization in Cerebral Venous Thrombosis., 49, 1828-1835 https://doi.org/10.1161/STROKEAHA.118.022129 (2018).

21. Kuroiwa, Y. et al. MR signal change in venous thrombus relates organizing process and thrombolytic response in rabbit. Magn Reson Imaging, 29, 975-984 https://doi.org/10.1016/j.mri.2011.04.015 (2011).

22. van Dam, L. F. et al. Magnetic Resonance Direct Thrombus Imaging (MRDTI) Can Distinguish Between Old and New Thrombosis in the Abdominal Aorta: a Case Report. Eur J Case Rep Intern Med, 7, 001351 https://doi.org/10.12890/2020_001351 (2020).

23. Moody, A. R. Magnetic resonance direct thrombus imaging. J Thromb Haemost, 1, 1403-1409 https://doi.org/10.1046/j.15387836.2003.00333.x (2003).

24. Phinikaridou, A. et al. In vivo magnetization transfer and diffusion-weighted magnetic resonance imaging detects thrombus composition in a mouse model of deep vein thrombosis. Circ Cardiovasc Imaging, 6, 433-440 https://doi.org/10.1161/CIRCIMAGING.112.000077 (2013).

\section{Figures}



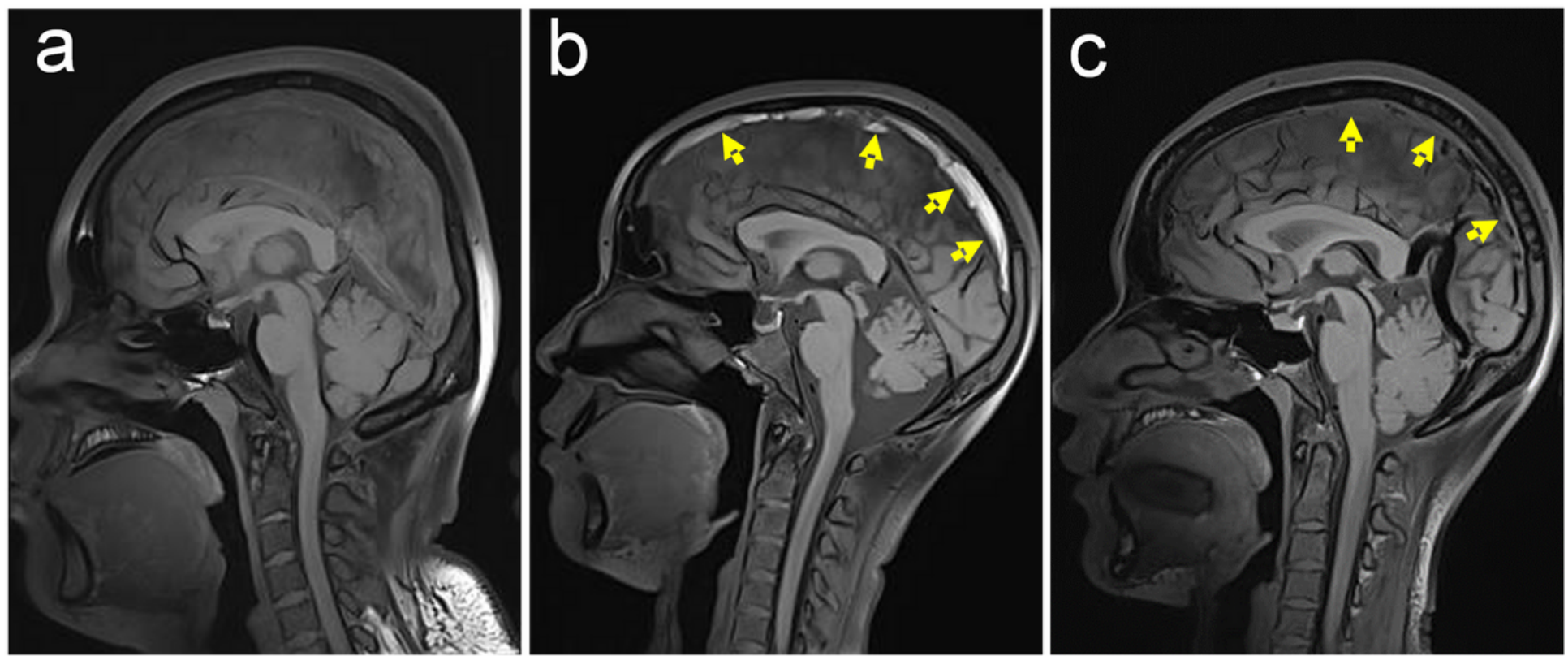

Figure 1

The thrombi (yellow arrows) at acute (a, 6days), subacute (b, 13 days), and chronic stages (c, 25 days) were typically depicted as isointense signals, hyperintense signals, and isointense signals, respectively. Compared to the thrombus at acute stage, the thrombus at chronic stage is organized and contractive.
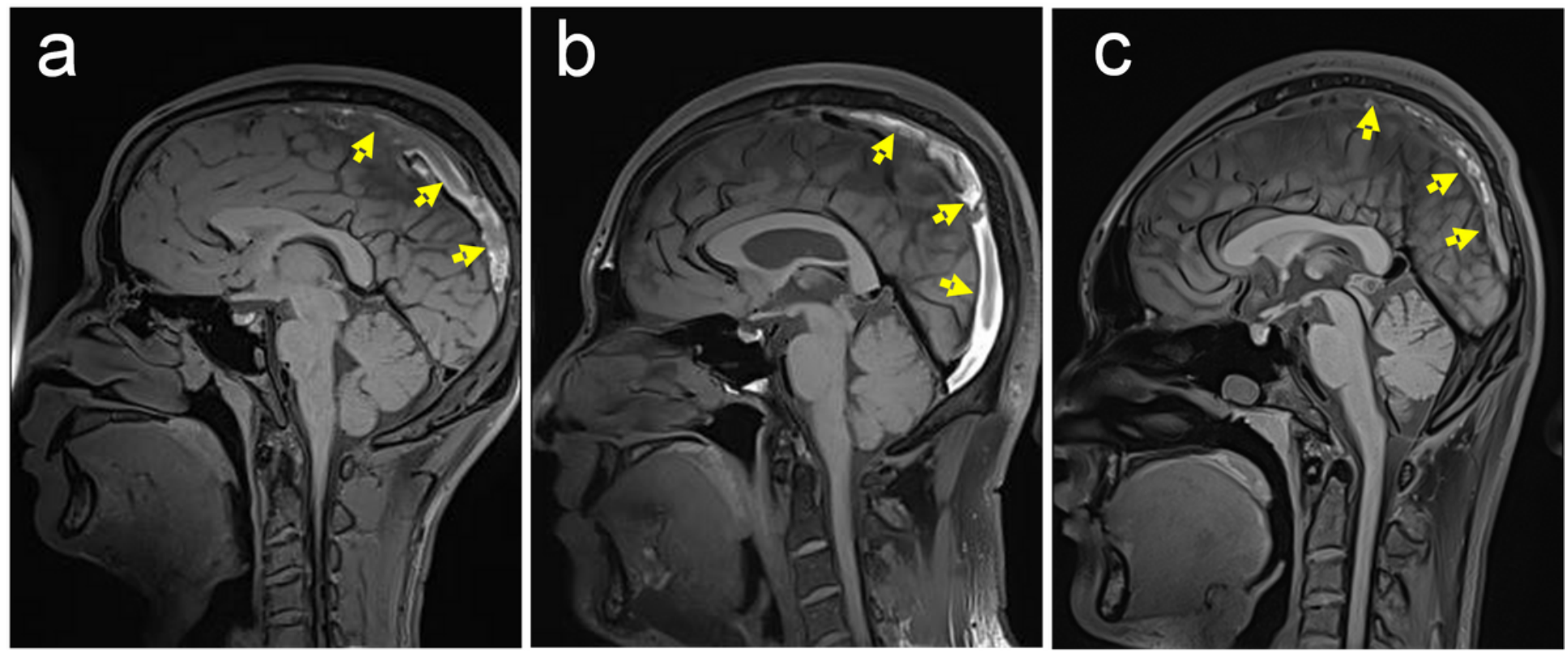

\section{Figure 2}

The thrombi (yellow arrows) presented in both isointense and hyperintense signals can be found in acute (a, 5 days), subacute (b, 10 days), or chronic CVST patients (c, 28 days), which should be at the transition period of acute-to-subacute or subacute-to-chronic stage. 


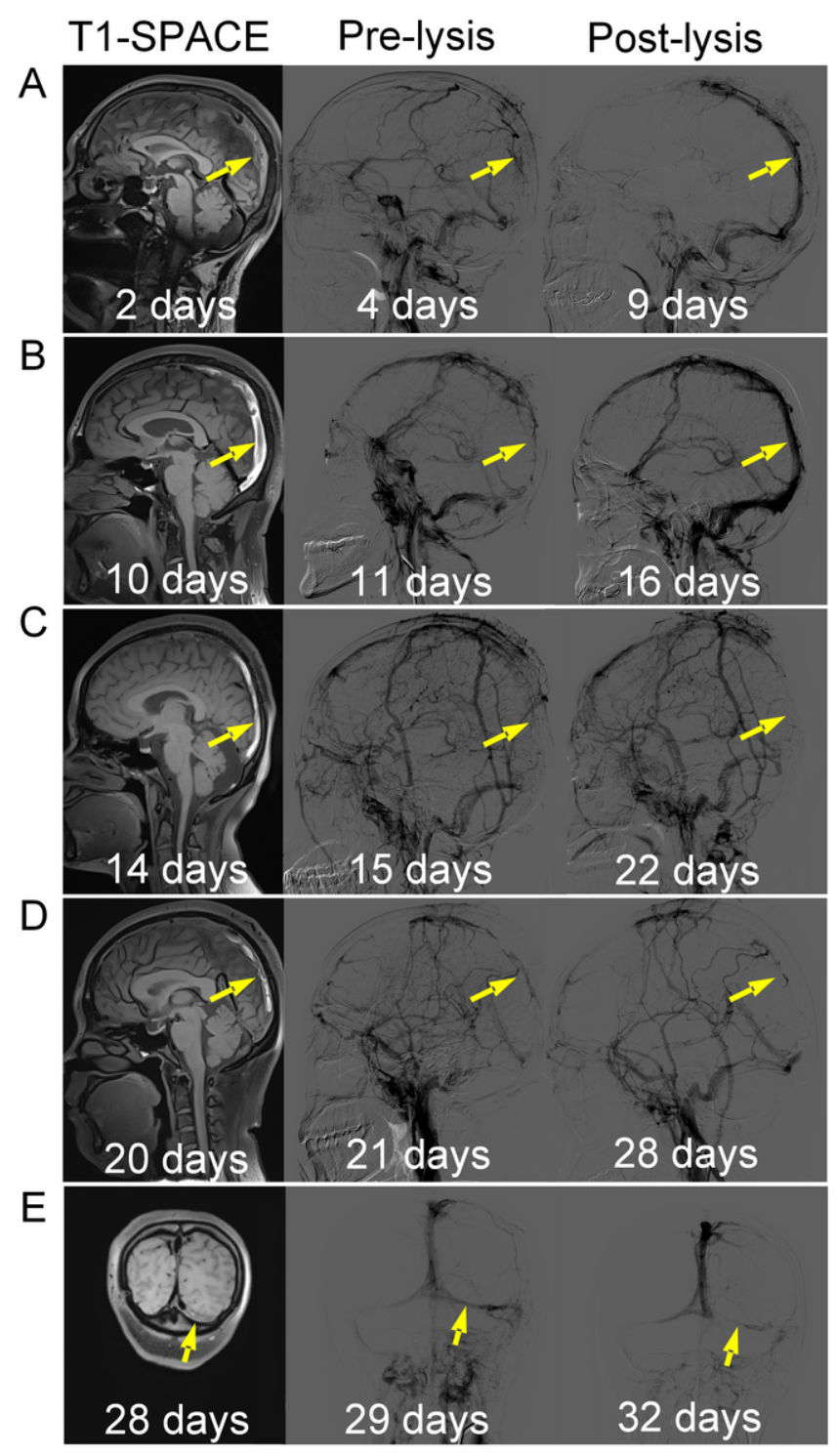

Figure 3

Recanalization of five CVST patients at different stages. According to the DSA study result, the thrombi at acute stage (A) and at the transition period from acute to subacute stage $(B, C)$ were completely dissolved; in contrast, the thrombi at the transition period from subacute to chronic stage (D) and at chronic stage (E) were not dissolved well. 

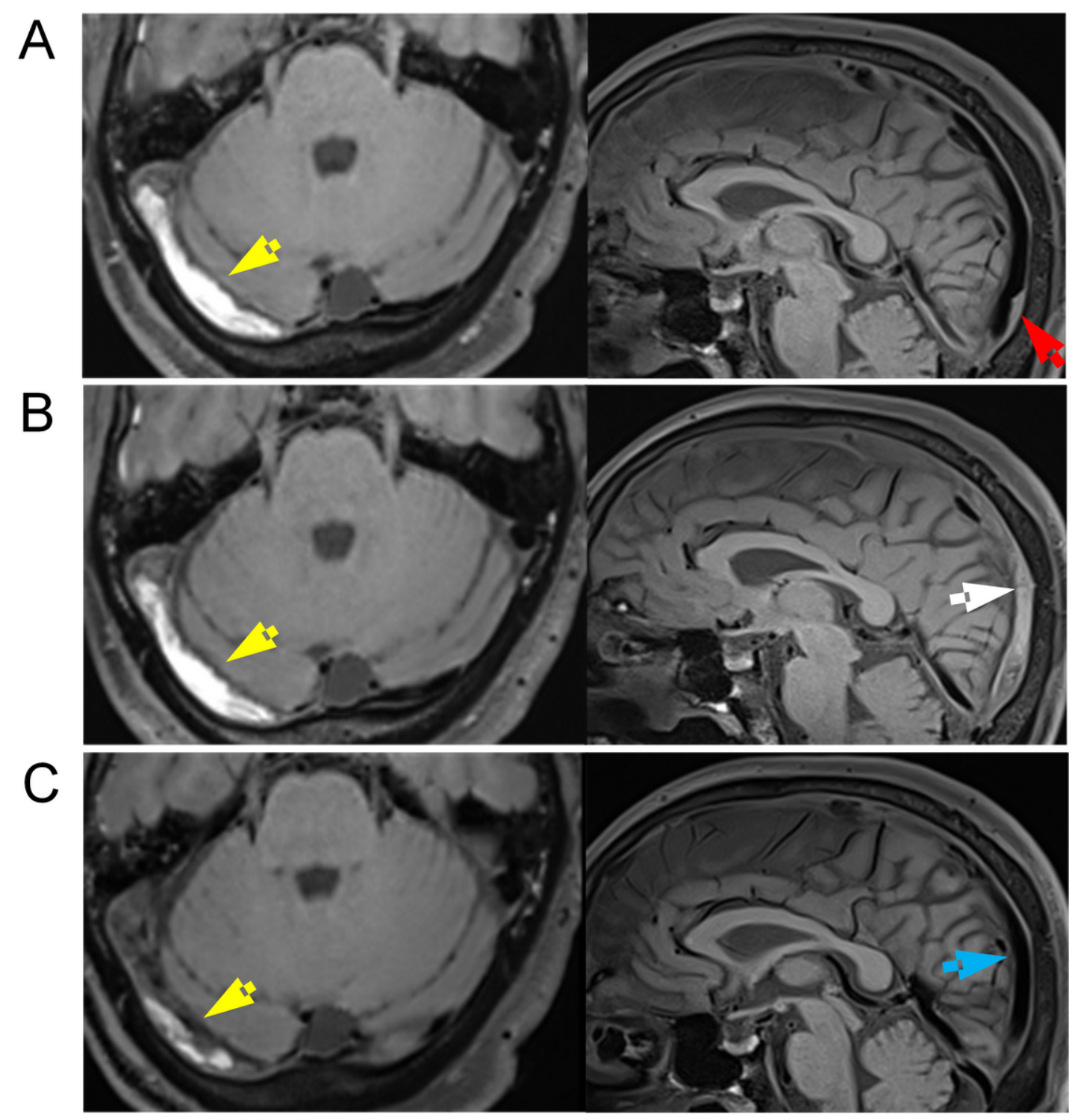

\section{Figure 4}

Images obtained from the patient with MR scan three times. Thrombus (yellow arrow) with hyperintense signals was found on the transverse sinus at 11 days after clinical symptom onset $(A)$. And there was only a very small thrombus with isointense signals ( $A$, red arrow) within the superior sagittal sinus. Two days later (the 13th days), the thrombus developed and fulfilled the whole superior sagittal sinus (B, white arrow). The newly developing thrombus $(B$, white arrow) presented in isointense signals should be at acute stage. After endovascular therapy at the 13th days, the acute thrombus (C, blue arrow) was successfully dissolved while the thrombus (C, yellow arrow) with hyperintense signals was residual at the 27 th days follow-up. 